\title{
ELEMENT AND ISOTOPIC FRACTIONATION IN CLOSED MAGNETIC STRUCTURES
}

\author{
T. H. ZURBUCHEN, L. A. FISK, G. GLOECKLER* and N. A. SCHWADRON \\ Department of Atmospheric, Oceanic and Space Sciences, University of Michigan, Ann Arbor, USA
}

\begin{abstract}
Recent papers have suggested that the slow solar wind is a super-position of material which is released by reconnection from large coronal loops. This reconnection process is driven by large-scale motions of solar magnetic flux driven by the non-radial expansion of the solar wind from the differentially rotating photosphere into more rigidly rotating coronal holes.

The elemental composition of the slow solar wind material is observed to be fractionated and more variable than the fast solar wind from coronal holes. Recently, it has also been reported that fractionation also occurs in ${ }^{3} \mathrm{He} /{ }^{4} \mathrm{He}$. This may be interpreted in the frame-work of an existing model for fractionation on large coronal loops in which wave-particle interactions preferentially heat ions thereby modifying their scale-heights.
\end{abstract}

Key words: Solar wind, solar wind composition, FIP fractionation, ${ }^{3} \mathrm{He} /{ }^{4} \mathrm{He}$ fractionation, solar magnetic field

\section{Introduction}

In this paper we discuss a recent model for fractionation processes occurring in the low corona. This model is strongly motivated by recent in situ and remote sensing observations. The in situ obscrvations have demonstrated that the elemental and isotopic composition in the solar wind strongly depend on the actual solar wind regime observed. Remote solar observations, e.g., from the SOlar and Heliospheric Observatory (SOHO), show that these compositional differences are also seen low in the solar atmosphere. It has been suggested that the elemental fractionation process occurs in the chromosphere (see, e.g., Geiss, 1982) where the solar material is being ionized. Judge et al. (1998) discuss recent composition observations in the chromosphere. Abundances in the solar wind are observed to be highly variable in time and they exhibit structures on very small spatial scales. This renders some of the time-stationary chromospheric fractionation mechanisms (see, e.g., Marsch et al., 1995; Peter, 1998) unlikely candidates. The first of these models also does not naturally imply a difference in composition patterns in the fast, coronal hole associated wind compared to slow solar wind. A fractionation theory is needed which does not strongly depend on the geometrical properties of the separating region and can naturally account for composition differences between fast and slow wind.

We introduce a new concept by first considering the overall picture of fast and slow solar wind in the heliosphere, at least near solar minimum, when the concepts for the overall structure are well developed: At high heliographic latitudes, the polar coronal holes give rise to a fast, $\sim 750 \mathrm{~km} / \mathrm{s}$ flow, which is remarkably steady

Also at Department of Physics and IPST, University of Maryland, College Park, USA

Space Science Reviews 85. 397-406, 1998.

(c) 1998 Kluwer Academic Publishers. Printed in the Netherlands. 


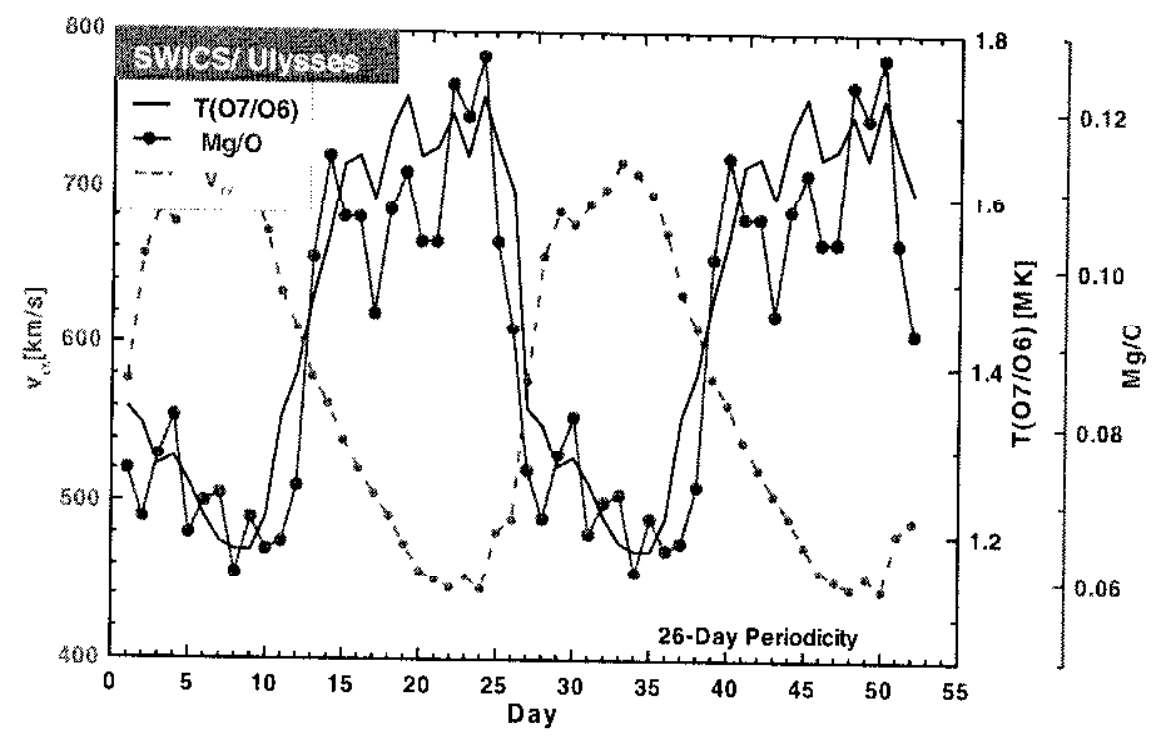

Figure 1. Superposed epoch analysis of Ulysses data showing the systematic variation in the $\mathrm{Mg} / \mathrm{O}$ ratio, the solar wind speed, and the coronal temperature inferred from the $\mathrm{O}^{7+} / \mathrm{O}^{6+}$ ratio during the (sidereal) solar rotation period. The abrupt transition of freeze-in temperatures and composition indicate a different origin for fast and slow wind. Figure adapted from von Geiss et al. (1995b).

(e.g., Phillips et al., 1995). However, at low latitudes, surrounding the streamer belt, the flow is slower, $\sim 400 \mathrm{~km} / \mathrm{s}$, but also more variable in density and speed (e.g. (josling, 1996).

The most interesting difference between fast and slow wind is clearly associated with the elemental abundances and charge state ratios as for example measured hy Ulysses-SWICS. A key observation from a paper by von Geiss et al. (1995b) is reproduced in Figure 1. Shown here are the results of a superposed epoch analysis of Ulysses-SWICS data showing the solar wind speed (plotted here is the spced the $\alpha$-particles which is the same as the proton speed to within an Alfven speed) and the $\mathrm{Mg} / \mathrm{O}$ ratio. $\mathrm{Mg}$ has a low first ionization potential (FIP) and is therefore casicr to ionize than $O$. Also plotted is the so-called freeze-in temperature $T_{O}$ of the solar wind which is a measure for the electron temperature about $>1-2$ solar radii from the photosphere. This temperature is typically around $1.5 \cdot 10^{6} \mathrm{~K}$ and highly variable when the solar wind is slow, and around $1.2 \cdot 10^{6} \mathrm{~K}$ in fast solar wind. Equivalently, the $\mathrm{Mg} / \mathrm{O}$ abundance ratio is fractionated in slow solar wind to become $\sim 0.11$, and close to photospheric in the fast solar wind. The steep transitions between the two solar wind types has been interpreted by Geiss et al. (1995a) to indicate a clear difference in the origin of fast and slow wind streams. These observations would he very hard to explain in a theory where coronal holes were the source of both fast and slow solar wind (see, e.g., Bravo et al., 1997).

We will first introduce a global theory for the magnetic configuration of the low corona and then relate slow solar wind observations to loop properties in the low 
latitude corona. A recent observational result will then be described which shows the spatial dependence of the ${ }^{3} \mathrm{He} /{ }^{4} \mathrm{He}-$ ratio in the solar wind. In section 3, this will then be interpreted in the framework of the theory described.

\section{Theory for the Slow Solar Wind and its Element Fractionation}

First, consider a theory for the origin and of the slow solar wind and its relation to the solar global magnetic field configuration. This theory is a natural consequence of the new concept for the heliospheric magnetic field in fast solar wind proposed by Fisk (1996), which is, that the footpoints of the heliospheric magnetic field move on the solar wind source surface. This motion results from an interplay between the differential rotation of the photosphere and the non-radial expansion of the solar wind into more rigidly rotating coronal holes.

The theory was motivated by the Ulysses observation of low-energy CIR-modulated particles which were observed up to very high heliospheric latitudes (Simnett et al., 1995). Fisk (1996) interpreted these observations to be clear indications for direct magnetic connections from low to high heliospheric latitudes. Such connections are not possible in a standard Parker magnetic field configuration, but they are a natural consequence of the new field configuration which results in magnetic field tiansport in the conona, leading to magnetic field connections of different heliospheric latitudes.

In Figure 2 the characteristics of this magnetic field transport from high to low latitude are shown (for details, see Fisk et al., 1998b, or Schwadron et al., 1998). The time-scale associated with this transport is of the order $\tau \simeq 1 / \omega \sim 100$ days, since $\omega=\Omega_{\text {equ }}-\Omega_{\text {pole }}$, and $\Omega_{\text {equ }}\left(\Omega_{\text {pole }}\right)$ is the equatorial (polar) rotation rate. On this time-scale the entire high-latitude magnetic flux is dumped into the low latitude regions. In Zurbuchen et al. (1997) direct observational evidence in support of this theory was presented. It is pointed nut that the high-latitude magnetic field as measured by Ulysses shows signatures of this transport, which are very hard to explain with a standard solar magnetic field model. If this high-latitude transport is really present, Maxwell's equations imply a transport at low latitudes. A natural mechanism for such a transport is reconnection, as is illustrated conceptually in Figure 2. This reconnection process releases material from previously closed magnetic structures such as loops. It can readily be demonstrated, as is discussed in Fisk et al. (1998b), that the typical properties of this transport is fully determined by the high latitude magnetic field configuration. For details concerning this magnetic field transport and reconnection scenario refer to Fisk et al. (1998b).

In this theory, there are therefore distinct regions from where solar wind can emerge (see Fisk et al., 1998a): First, it can be emitted along continuously open magnetic field lines in coronal holes, yielding the fast steady wind. On the other hand, slow solar wind emerges from closed magnetic field regions, which are continuously opencd by reconnection of what had been closed magnetic structures. 


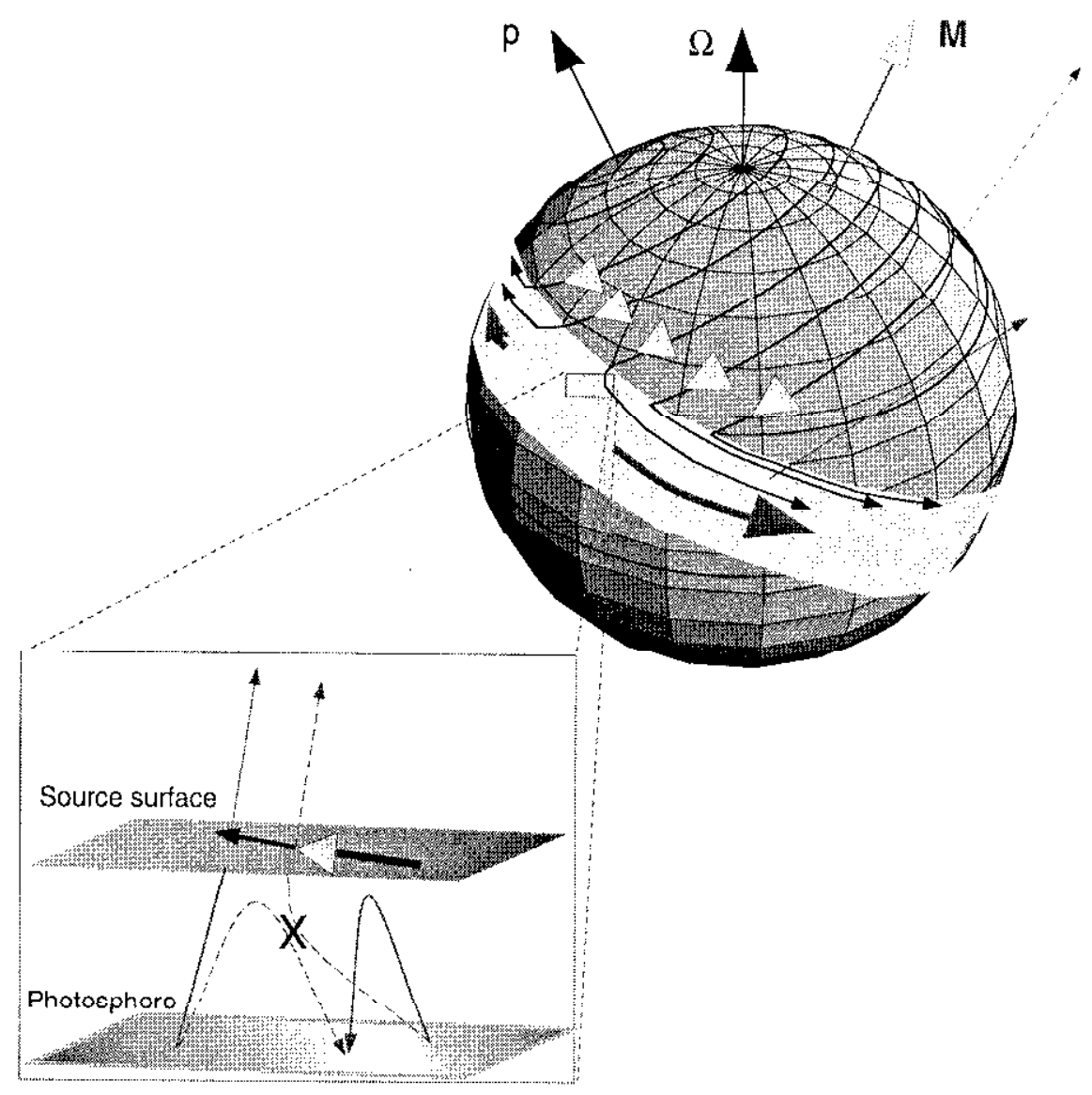

Figure 2. Reconnection scenario as described in the text. Shown is the source surtace of the solar wind at about 2 solar radii in a frame co-rotating with the equatorial rotation rate. In open magnetic field regions, close to the pole, footpoints move in latitude. They are eventually convected into the band of closed magnetic fields at low latitudes. Due to sub-source surface reconnection events, a diffusive transport in longitude closes the footpoint curves on the solar wind source surface.

This makes the slow solar wind a time-dependent flow of plasma which is fed from many distinct sources. The compositional signatures of the slow solar wind are therefore closely related to the physical properties of the magnetic loops from where the wind emerges.

Recently, data from the SWICS-ACE instrument (for instrument descriptions see, Gloeckler et al., 1998b) has been presented by Hefti et al. (1998) which is consistent with the framework described above. Figure 3 shows composition measurements of solar wind with a time-resolution of 13 minutes during a period of five days. During day 92 there are signatures of a coronal mass ejection event, afterwards there is a period of standard slow solar wind. Large fluctuations of the $\mathrm{O}$ charge state ratio $n_{7} / n_{6}$ and also of the $\Gamma \mathrm{e} / \mathrm{O}-\mathrm{ratio}$ are visible through out the 


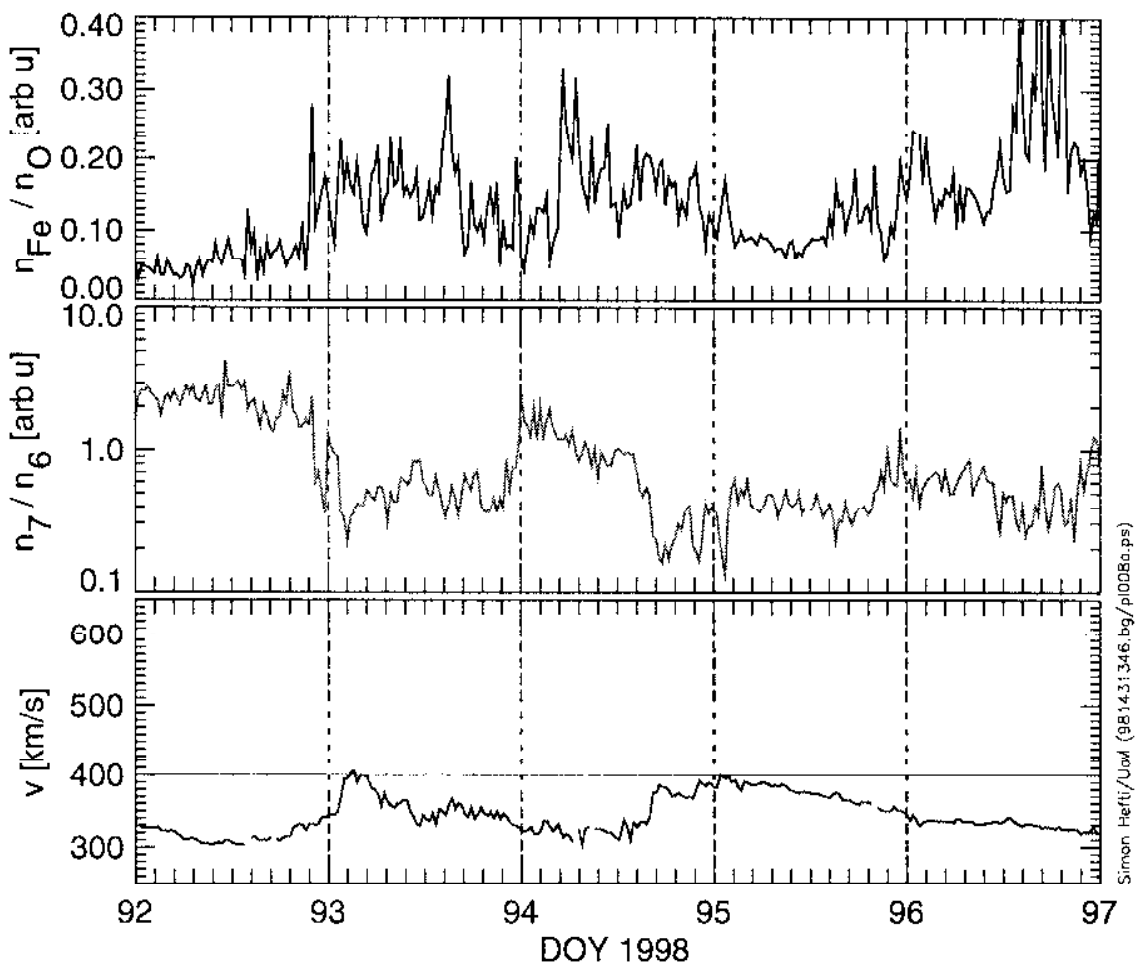

Figure 3. Compositional variations within the slow solar wind observed with ACE/SWICS. The O charge state ratio $\mathrm{O}^{7+} / \mathrm{O}^{6+}$ and the $\mathrm{Fe} / \mathrm{O}$ ratio show clear variations. Notice that there are correlated changes of the two parameters which are occurring on a time-scale of days and fractions of a day. Figure from Hefti et al. (1998).

entire period. Notice also there are clear correlated changes of the two parameters on time-scales of days or fractions of a day. The charge state ratio $n_{7} / n_{6}$ is determined in the corona around temperatures of $1.5 \cdot 10^{6} \mathrm{~K}$. On the other hand, the Fe/O-ratio is variable depending on a FIP-fractionation which has to occur in a temperature range of $5 \cdot 10^{4} \mathrm{~K}$ (Geiss, 1982). This result, in accordance with the model described above, is consistent with the presence of closed magnetic structures for slow solar wind and element fractionation in the solar wind.

There are several possibilities for modeling a FIP-effect in closed magnetic structures. One simple mechanism is discussed in detail by Schwadron et al. (1998) who considers the effects of wave-heating of particles in closed magnetic structures. Similar to the in situ observations, heavy ions in the corona are not observed to have equal temperatures hit are non-thermally heated, presumably by magnetic waves (see Kohl et al., 1997). Schwadron et al. (1998) considered the effects of such wave-particle interactions in loops. They concluded that if MHD-waves propagate sufficiently far down into the "legs" of the loops in hydrostatic equilib. 
rium, a FIP fractionation naturally occurs since the scale-height of a given species id highly dependent on the degree to which it is ionized. With this simple model, Schwadron et al. (1998) managed to reproduce the average fractionation pattern observed in the slow solar wind very well.

The mechanism in this theory depends on the presence of long-lived large coronal loops on which hydrostatic equilibrium can be achieved. In coronal holes, loops are much smaller and fractionation does not work as efficicntly. Therefore FIP enhancements in coronal hole associated wind are not expected. It should also be noted that the FIP enhancements in this theory are highly dependent on the field configurations in the low corona. That is, composition differences are expected in regions with differing field configurations.

\section{3. ${ }^{3} \mathrm{He} /{ }^{4} \mathrm{He}$ Fractionation in Loops}

Gloeckler and Geiss (1998) recently reported observations of the ${ }^{3} \mathrm{He} /{ }^{4} \mathrm{He}-$ ratio $\left(\Xi_{\mathrm{He}}\right.$ ) ratio by SWICS-Ulysses over a time-period of almost 3000 days. The result can be sumnarized as follows:

1. In the low latitude solar wind, $\Xi_{\mathrm{He}}$ is $(4.08 \pm 0.25) \cdot 10^{-4}$. This is consistent with previous in situ measurements.

2. $\Xi_{\mathrm{He}}$ varies systematically in accordance to the FIP fractionation processes: If the elemental abundances are close to photospheric (e.g., in the fast solar wind), $\Xi_{\mathrm{He}}$ is smaller. During periods in which low FIP ions are not enhanced, $\Xi_{I I}$ is $(3.16 \pm 0.25) 10^{-4}$.

It has been pointed out by Gloeckler and Geiss (1998) that the value of $\Xi_{\mathrm{He}}$ determined in situ is a very reliable source for the $\mathrm{Hc}$ isotopic ratio in the outer convective zone. It is therefore important to understand what value of $\Xi_{\mathrm{He}}$ is most representative for the solar surface, or, in other words, how ${ }^{3} \mathrm{He}$ is fractionated relative $10{ }^{1} \mathrm{He}$. In the following subsections we will describe a model which will show that the $\Xi_{\mathrm{He}}$ in the solar wind turns out to be an upper limit for the value of $\Xi_{\mathrm{He}}$ in the outer convective zone.

In the following subsections we will argue that the solar wind value of $\Xi_{\mathrm{He}}$ is an upper limit for the solar value of interest. Using a simple approximation of the Schwadron et al. (1998) process we will show that ${ }^{3} \mathrm{He}$ can be enriched relative to ${ }^{4} \mathrm{He}$ in closed magnetic structures and a qualitative dependence of $\Xi_{\mathrm{He}}$ and the FIP fractionation is predicted.

\subsection{THE MODEL}

Consider a loop in hydrostatic equilibrium. The neutrals are given by

$$
\frac{\partial}{\partial z} P_{s n}=-\rho_{s n} g+\nu_{s u}\left(u_{s n}-u\right)
$$


where $z$ is the distance along the magnetic field, $P_{s n}$ is the pressure; $\rho_{s n}$ the mass density of the neutral particles of species $s$. Solar gravity is denoted by $g$, and $u$ is the velocity of hydrogen. The neutrals are coupled to hydrogen by collisions with frequency $\nu_{s n}$. The pressure of neutral atoms is given by

$$
P_{s n}=\frac{\rho_{s n}}{2}\left(c_{s}^{2}+v_{T}^{2}\right),
$$

where as $c_{s}$ is the speed of sound and $v_{T}$ is the turbulent velocity of the plasma.

For ions, the equations look slightly different:

$$
\frac{\partial}{\partial z} P_{s i}=-\rho_{s i} g+\left(\nu_{s i}+\nu_{s w}\right)\left(u_{s i}-u\right)
$$

The coupling to hydrogen occurs not only due to collisions but also because of wave-particle interactions given by $\nu_{s w}$. This interaction also affects the pressure equation in the form of $v_{W}$.

$$
P_{s i}=\frac{\rho_{s i}}{2}\left(c_{s}^{2}+v_{T}^{2}+v_{W}^{2}\right)
$$

(See Schwadron et al. (1998) for a detailed discussion of these terms.) To solve this set of equations, a temperature and He charge state profile for the solar atmosphere must be assumed. In order to include the non-local effect of photo-ionization, a numerically determined atmosphere (VAL-C) by Vernazza et al. (1981) has been used here. We also assume $u=0$, in agreement with the assumptions of Vernazza et al. (1981).

The wave particle interaction of ${ }^{4} \mathrm{He}$ and ${ }^{3} \mathrm{He}$ is qualitatively and quantitatively different. It has been known for a long time that ${ }^{3} \mathrm{He}$ can be enhanced in impulsive particle events. These enhancements are believed to occur due to resonant waveparticle interactions (see, e.g., Fisk, 1978, and Roth et al., 1997). These effects preferentially heat ${ }^{3} \mathrm{He}$ and therefore cause $\Xi_{\mathrm{He}}$ enhancements by several orders of magnitude above the solar value. These ratios are representative for particles above a threshold for acceleration. They originate therefore from the high energy tails of the distribution functions. However, also the thermal part of the distribution function is slightly heated if these iesontant wave-particle effects occur in the low corona. This increased effective temperature (or $v_{W}$ in equation 4) of ${ }^{3} \mathrm{He}$ will result in a larger scale-height and therefore, $\Xi_{\mathrm{He}}$ enhancements will occur. To demonstrate this, we integrate equations (1) and (3) using the atmosphere model from Schwadron et al. (1998) and the wave-heating terms mentioned there. In order to model the preferential heating, we enhance $v_{W}$ for ${ }^{3} \mathrm{He}$ by a small amount relative to the value used for ${ }^{4} \mathrm{He}$.

\subsection{RESULTS}

Low down in the loop, where He is mostly neutral, the turbulent velocity $v_{T}$, is dominantly affecting the pressurc. Therefore, no significant mass fractionation occurs. 


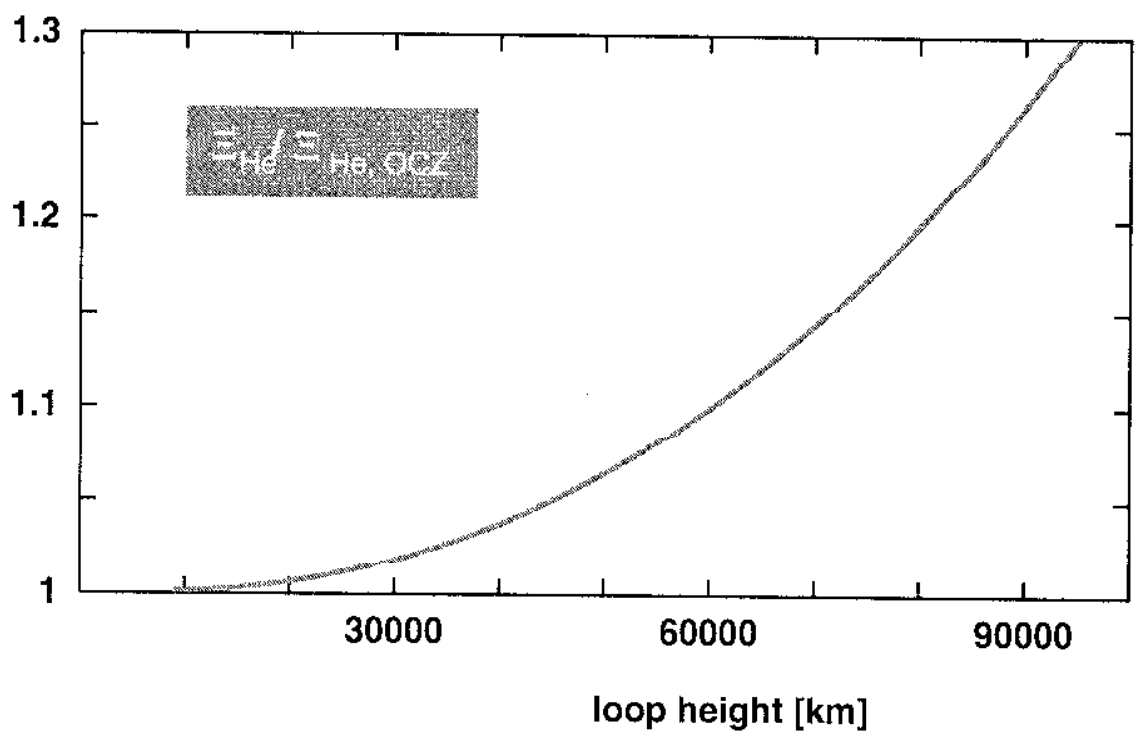

Figure 4. Loop height dependence of the He isotopic ratio, $\Xi_{\text {He }}$, in a wave-heated loop with preferential heating of ${ }^{3} \mathrm{Hc}$. The heating of ${ }^{3} \mathrm{I}$ le is assumed to be $0.2 \%$ nore efficient than the ${ }^{4} \mathrm{He}$ healing. The relative enhancement is dependent on the loop height.

Further up in the transition region, He gets ionized and therefore starts interacting with the wave-field in the loop. If ${ }^{3} \mathrm{He}$ was heated the same as ${ }^{4} \mathrm{He}$, the pressure given by equation (4) would be determined by the wave-heating term $v_{W}$ and therefore, again, almost no fractionation occurs.

Because of the fact that the heating of ${ }^{3} \mathrm{He}$ is additionally affected by resonant wave-heating, a slightly larger $v_{W}$ is expected for ${ }^{3} \mathrm{He}$ leading to fractionation, enhancing $\Xi_{\mathrm{He}}$ relative to its value in the outer convective zone. This is illustrated in Figure 4. For a very modest enhancement of the wave particle interaction of ${ }^{3} \mathrm{Hc}$ rclative to ${ }^{4} \mathrm{IIe}$ by $0.2 \%$, the abundance fractionation of the lighter ${ }^{3} \mathrm{He}$ isotope becomes significant. The enrichment of ${ }^{3} \mathrm{He}$ relative to ${ }^{4} \mathrm{He}$ is also correlated with the actual loop length. This same loop length dependence naturally also holds for

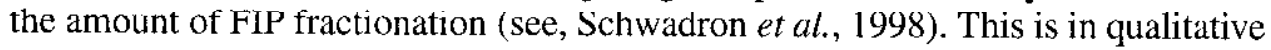
agreement with the observational result by Gloeckler and Geiss (1998) described above.

According to this study, $\Xi_{\mathrm{He}}$ measurements in the solar wind are only upper limits on $\Xi_{\mathrm{He}}$ and the coronal hole value $\Xi_{\mathrm{He}}=(3.16 \pm 0.25) \cdot 10^{-4}$ is the best estimate for the He isotope ratio in the onter convective zone.

\section{Concluding Remarks}

We have considered the consequences of the new heliospheric magnetic field mod$\mathrm{cl}$ on the sources of the solar wind. Because of the lange-scale tansport of Indantetic 
flux as described by Fisk (1996), reconnection is systematically forced at low latiudes. These reconnection events result in an intennitlent source of plasma wlich was previously confined in closed loops, the slow solar wind.

The elemental composition of solar wind plasma is then strongly dependent on the actual source in the low corona, which is of course intimately linked to the chromosphere, where the first ionization occurs. The focus of the study of fractionation effects should therefore be not only on the chromosphere, the actual location of the first ionization, but also on the transition region and the low corona, where the plas$\mathrm{ma}$ is undergoing wave-heating and gravitational settling. Unfortunately, the direct experimental test of these effects is very difficult, since all nptical ohservations of line-of-sight integrals are dominated by structures of high number density. Large, low density loops would probably be more likely candidates for the source of the slow solar wind.

We should also remember that the theory described here applies only in the years around solar minimum, when there are well-developed polar coronal holes and a very distinct region of magnetically closed topology. It is not clear how this mechanism will apply near solar maximum. There are certainly coronal holes on the Sun nearer to solar maximum, but they are short lived, and the concept of field line motion across them, with resulting reconnection in closed loops, may be quite different.

\section{Acknowledgements}

The work was supported, in part, by NASA contract NAS5-32626 and NASA/JPL contract 955460. T.H.Z. and N.A.S. were also supported, in part, by an NFS grant ATM 9714070. We thank S. Ilefti, R. von Stciger, E. H. Avrett, and C. Pei for many useful discussions.

\section{References}

Bravo, S., and Stewart, G. A.: 199/, 'Fast and slow solar wind from sulal com unal lioles', Astrophys. J. 489,992 .

Fisk, L. A.: 1978, '33e-rich flares: A possible explanation', Astrophys. J. 224, 1048.

Fisk, I. A.: 1996. 'Motion of the footpoints of heliospheric magnetic field lines at the sun: Implications for recurrent energetic particle cvents at high heliographic latitudes', J. Geophys. Res. 101, 15547.

Fisk, L. A., Schwadron, N. A., and Zurbuchen, T. H.: 1998a, 'On the slow solar wind', Space Sci. Rev., in pless.

Fisk, L. A., Zurbuchen, T. H., and Schwadron, N. A.: 1998b, 'On the slow solar wind: I. Origin in the coronal magnetic ficld', Astrophys. J., in press.

Geiss, J.: 1982, 'Processes affecting abundances in the solar wind', Space Sci. Rev. 33, 201.

Geiss, J., et al:: 1995a, 'The southern high-speed stream: Results trom the swics instrument on Ulysses', Science 268, 1033.

Geiss, J., Gloeckler, G., and von Steiger, R.: 1995b, 'Origin of the solar wind from composition data', Space Sci. Rov 72, 19. 
Gloeckler, G., and Geiss, J.: 1998, "Measurement of the abundance of helium-3 in the Sun and in the local interstellar cloud with SWICS on Illysses', in Primordial Nuclei and Their Calactic Evolution, Eds. N. Prantzos, M. Tosi and R. von Steiger, Kluwer, 275.

Gloeckler, G, Bedini, P., Fisk, L. A., Zurbuchen, T. H., Ipavich, F. M., Cain, J., Tums, E. O., Bochsler, P., Fischer, J., Wimmer-Schweingruber, R. F., Gciss, J., and Kallenbach, R.: 1998, 'Investigation of the composition of solat and inter stellar malter using solar wind and pickup ron measurements with SWICS and SWIMS on the ACE spacecraft', Space Sci. Rev., in press.

Gosling, J. T., 1996: 'Physical nature of low-speed solar wind', in Robotic Exploration close to the Sun: Scientific Basis, AIP Conference Proc., 385.

Hefti, S., Zurbuchen, T. H., Fisk, L. A., Gloeckler, and G., Schwadron, N. A., 1998: 'Compositional variations in the slow solar wind: ACE/SWICS results', EOs Trans. AGU, Spring Meet. Suppl., S259.

Judge, P. G., and Peter, H.: 1998, 'The structure of the chromospherc', Space Sci. Rev., this volunte.

Kohl, J. L. et al:: 1997, 'First results from the solar ultraviolet coronagraph spectrometer', Solar Phys. 175, 613.

Marsch, E., von Steiger, R., and Bochsler, P.: 1995, 'Element fractionation by diffusion in the solar chromosphere', Astron. Astrophys. 301, 261.

Peter, H.: 1998, 'Element separation in the chromosphere', Space Sci. Rev., this volume.

Phillips, J. L., et al.: 1995, 'Ulysses solar wind plasma observations at high southerly latitudes', Science 268. 1030.

Roth, I., and Temerin, M.: 1997, 'Enrichment of ${ }^{3} \mathrm{He}$ and heavy ions in impulsive solar flares', Astrophys. J. 477, 940.

Schwadron, N. A., Fisk, L. A., and Zurbuchen, T. H.: 1998, 'On the slow solar wind: II. Element fractionation', Astrophys., J., iu pless.

Simnetı, G. M., Sayle, K. A., Tappin, S. J., and Roelof, E. C.: 1995, 'Corotating particle enhancements out of the ecliptic plane', Space Sci. Rev. 72, 327.

Vernazza, J., Avrett, E. H., and Loeser, R.: 1981: ApJS 45, 635.

Zurbuchen, T. H., Schwadron, N. A., and Fisk, L. A.: 1997, 'Direct observational evidence for a heliospheric magnetic field with large excursions in latitude', J. Geophys. Res. 102, 24175.

Address for correspondence: Thomas $\mathrm{H}$. Zurbuchen, Space Research Laboratory, University of Michigan, 2455 Hayward Street, Ann Arbor, MI 48109-2143 